\title{
Point de vue médical sur le suicide assisté - la bienveillance négligée
}

\section{Gian Domenico Borasio}

Prof., Service de soins palliatifs, Centre Hospitalier Universitaire Vaudois, Lausanne

\section{Il devrait être possible de proposer des aides concrètes aux personnes qui se} trouvent dans des situations désespérées. Toutes les tentatives de légiférer au niveau fédéral en matière d'assistance au suicide ont échoué. Dans cette thématique ultrasensible, le législateur devrait veiller à ne pas renforcer unilatéralement l'aspect de l'autonomie (qui est à présent de facto délégué aux associations d'aide au suicide); la bienveillance devrait enfin recevoir la reconnaissance législative qui lui est due.

\begin{abstract}
On assiste actuellement, dans l'ensemble de l'Europe, à un vif débat autour de la fin de vie. Aussi bien en Angleterre qu'en France, en Allemagne et en Autriche, les politiciens et les représentants de diverses visions du monde et professions discutent de manière intense sur de possibles modifications de la pratique légale en vigueur dans leur pays. Dans ce contexte, le rôle que peut jouer le corps médical dans la préparation, la réalisation et/ou l'accompagnement de mesures d'aide au décès (euthanasie active ou suicide assisté) est particulièrement controversé.
\end{abstract}

On n'a pas répondu à la nécessité d'offrir une aide concrète aux personnes qui se trouvent dans des situations désespérées.

Le but du présent article est de fournir un bref aperçu des données empiriques existant sur l'euthanasie active (homicide sur demande de la victime) et le suicide assisté dans les principaux pays et régions qui autorisent ces pratiques. Sur cette base, quelques réflexions seront formulées sur l'évolution possible en Suisse et des arguments en faveur d'une législation fédérale seront soumis à la discussion.

\section{Données empiriques}

Si l'on considère les chiffres provenant des Etats qui autorisent l'euthanasie active et/ou le suicide assisté, il en ressort une image intéressante (ill.): dans les pays qui autorisent ces deux pratiques, c'est l'euthanasie active qui est réalisée beaucoup plus fréquemment. Aux Pays-Bas, 28 personnes sur 1000 sont décédées par euthanasie en 2012, mais seulement une seule personne sur 1000 par suicide assisté. En Belgique, la proportion de décès par suicide assisté est encore plus faible, d'après les estimations. Malgré une nette augmentation des cas d'euthanasie aux Pays-Bas et en Belgique ces dernières années, le suicide assisté reste rare dans ces deux pays.

La nette prépondérance des cas d'euthanasie active par rapport aux suicides assistés dans les pays où ces deux pratiques sont autorisées prouve que les patients préfèrent déléguer au médecin le geste mettant fin à leurs jours. Le fait que la fréquence des mesures raccourcissant la vie soit nettement plus élevée en Belgique et aux Pays-Bas par rapport aux régions dans lesquelles on n'autorise que le suicide assisté démontre que la possibilité de recourir à l'euthanasie active abaisse considérablement le seuil psychologique d'une demande d'aide au décès. Dans ce contexte également, il paraît justifié - et indiqué - de maintenir le caractère punissable de l'homicide sur demande de la victime.

La Suisse, qui tolère uniquement l'assistance au suicide sans pour autant légiférer en la matière, présente une ébauche de "courbe de croissance» similaire à celle de la Belgique et des Pays-Bas, même si le niveau reste plus faible. Cela pourrait s'expliquer par l'absence de règles procédurales et de garde-fous, d'autant plus qu'un nombre croissant de personnes ne souffrant pas d'une maladie physique ont recouru au suicide assisté en Suisse au cours des dernières années. En Oregon par contre, où l'aide au suicide n'est autorisée que pour les personnes gravement malades et de manière strictement réglementée, on constate seulement une augmentation marginale des cas de suicide assisté, avec une fréquence de moins d'un $10^{\mathrm{e}}$ par rapport à l'euthanasie active aux Pays-Bas. 


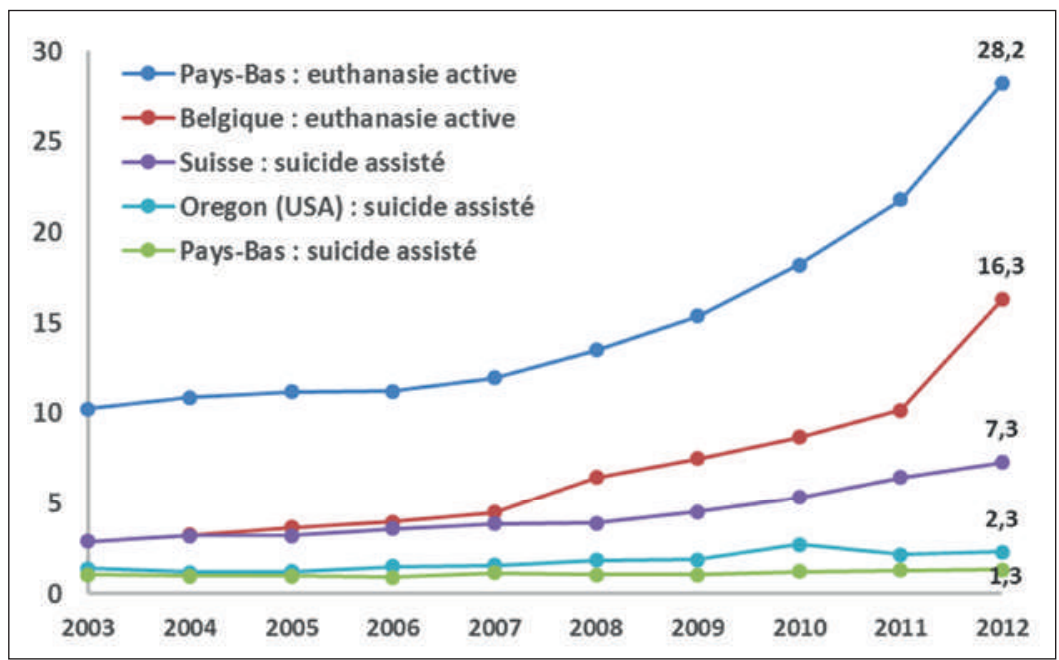

Incidence annuelle (sur 1000 décès) de l'euthanasie active (homicide sur demande de la victime) et du suicide assisté dans plusieurs pays (tiré de la référence 4).

\section{Réflexions sur l'évolution en Suisse}

L'auteur de cet article ne travaille en Suisse que depuis quatre ans et ne peut dès lors pas prétendre connaître de manière exhaustive les détails de l'évolution qui a eu lieu à ce jour. Si l'on considère ce qui se passe dans d'autres pays, il semble néanmoins concevable que l'élargissement de l'assistance au suicide aux vieillards et aux personnes souffrant de maladies chroniques comme le préconise désormais Exit [1] puisse générer des conséquences négatives. Un tel système pourrait, en raison de mécanismes économiques immanents, conduire progressivement à une situation dans laquelle ces personnes devraient se justifier de ne pas recourir à l'option du suicide assisté et de constituer ainsi une "charge» pour la collectivité. D’un point de vue médical, on ne peut que mettre en garde face à une telle évolution. Les vieillards et les personnes souffrant de maladies chroniques doivent souvent lutter contre une diminution de leur autonomie. Leur mobilité est généralement réduite, ils souffrent de manière croissante de déficits cognitifs et sensoriels (même si, d'un point de vue juridique, leur capacité de discernement est conservée). Ce n'est pas sans raison qu'on les qualifie de «fragiles». Ces personnes, que nous deviendrons nous-mêmes un jour, ont particulièrement besoin de l'aide et de la bienveillance de leur famille et de la société tout entière.

\section{Autonomie et bienveillance: le déséquilibre}

L'autonomie et la bienveillance se trouvent parfois dans une relation dichotomique, chaque patient nécessitant de son médecin un «mélange» très individuel de ces deux composantes que sont la bienveillance et le respect de sa liberté de choix. L'équilibre entre autonomie et bienveillance peut tout à fait varier au cours d'une maladie grave - en général plutôt dans le sens de la bienveillance. Aller chercher le patient toujours exactement là où il se trouve, c'est l'un des aspects centraux de l'art médical. Comme le dit le philosophe danois Søren Kierkegaard: «Il faut, pour vraiment aider quelqu'un, avoir tout d'abord soin de le prendre et de commencer là où il est. C'est le secret de la bienveillance. Quiconque en est incapable est dans l'illusion quand il croit pouvoir être utile à autrui. Pour aider vraiment quelqu'un, je dois être mieux informé que lui, mais tout d'abord je dois comprendre ce qu'il comprend, faute de quoi ma maîtrise ne lui est d'aucun profit» [2].

En Suisse, dans le domaine de l'assistance au suicide, l'Etat a laissé le champ de l'autonomie de facto (par manque d'action législative) aux organisations d'aide $\mathrm{au}$ suicide. Ces dernières travaillent la plupart du temps de manière très consciencieuse à l'intérieur du cadre fixé par le Code pénal suisse (art. 115 CPS) et le ministère public au niveau cantonal. Etant donné que ces organisations incarnent le principe de l'autodétermination, il n'est pas étonnant qu'elles aient tendance

\section{Aller chercher le patient là où il se trouve, c'est} l'un des aspects centraux de l'art médical.

à élargir toujours plus ces «limites», dernièrement avec leur intervention en faveur de l'assistance au suicide pour les vieillards. Sur le fond, il n'y a rien à objecter à cela, étant donné que l'activité des organisations d'assistance au suicide reflète une forte acceptation de ces pratiques par la société et un besoin marqué d'autodétermination. Mais qu'en est-il de l'autre pôle: la bienveillance?

De mon point de vue, la législation suisse a échoué dans ce domaine jusqu'à présent. Toutes les tentatives de légiférer sur l'assistance au suicide se sont soldées par un échec, ne permettant pas de répondre à la nécessité d'offrir une aide concrète aux personnes qui se trouvent dans des situations désespérées. La bienveillance est cependant un devoir central de l'Etat. L'une des affirmations essentielles de la Constitution fédérale est que «la force de la communauté se mesure au bien-être du plus faible de ses membres». Il est vrai que, ces dernières années, la prévention du suicide, de même que les soins palliatifs, ont été renforcés dans le cadre de programmes nationaux, partiellement avec succès. Toutefois, les personnes qui souffrent trop pour continuer à vivre considèrent généralement les organisations d'assistance au suicide comme leurs interlocu- 
teurs primordiaux. Et ces associations ne peuvent pas couvrir l'aspect de la bienveillance; elles n'en ont ni le mandat ni les compétences.

\section{Réflexions sur une proposition de loi}

Il existe une alternative à la léthargie législative en matière d'aide au suicide: l'exemple du canton de Vaud. La nouvelle loi cantonale qui est entrée en vigueur le 1.1.2013 après une votation populaire réglemente l'aide au suicide dans les hôpitaux et les EMS. Plusieurs obligations y figurent, notamment celles d'informer le médecin traitant et de proposer les aides existantes, en particulier la médecine palliative.

Malgré sa complexité incontestée, la loi a été acceptée par une large majorité, et cela à la place de la proposition initiale d'Exit A.D.M.D. qui était formulée de manière très simple et ne visait qu'à permettre un accès illimité à l'aide au suicide aux résidents des EMS. Le

\section{Une loi prévoyant que toute assistance au suicide doit être précédée d'une consultation par des médecins qualifiés constituerait une étape importante.}

résultat de cette votation démontre que la population est tout à fait capable de distinguer entre les initiatives paternalistes (visant à limiter la liberté de décision de l'individu) et celles qui sont portées par un esprit de bienveillance.

Faire preuve de bienveillance ne signifie pas retirer à quelqu'un son libre-arbitre, mais bien plutôt mettre cette personne dans une position où elle peut prendre une décision éclairée. Dans ce sens, une loi fédérale qui prévoirait que toute assistance au suicide doit être précédée d'une consultation par des médecins qualifiés (selon la situation: gériatres, psychogériatres, spécialistes en médecine palliative ou psychothérapeutes spécialement formés) constituerait une étape importante dans les efforts visant à redonner sa place au principe de bienveillance qui a été négligé jusqu'à présent.

\section{Le rôle du corps médical}

Le corps médical suisse, représenté par ses principaux organes, la FMH et l'ASSM, s'est penché à de nombreuses reprises et avec beaucoup de soin sur la thématique de l'assistance au suicide. Une étude récente (non représentative), réalisée en 2014 , révèle une attitude ambivalente: $78 \%$ des médecins ayant répondu considèrent que l'aide au suicide en fin de vie est fondamentalement défendable mais moins de la moitié seraient disposés à la fournir eux-mêmes [3].

Une révision des directives de l'ASSM est à l'ordre du jour. Une des questions centrales est de savoir s'il faut maintenir le principe selon lequel l'aide au suicide n'est pas une tâche médicale. Le débat promet d'être passionnant. Indépendamment de l'issue de celui-ci, de mon point de vue de médecin et de palliativiste, il est incontestable que le conseil sur les alternatives au suicide est une tâche essentielle du médecin - selon l'ancienne description de la profession médicale: "guérir parfois, soulager souvent, consoler toujours». Le législateur devrait veiller, dans cette thématique ultrasensible, à ne pas continuer à renforcer unilatéralement l'aspect de l'autonomie; il faudrait que l'aspect, tout aussi important, de la bienveillance bénéficie enfin d'une reconnaissance législative attendue depuis longtemps. Le corps médical peut - et devrait - jouer un rôle actif dans cette évolution.

\section{Références}

1 Exit deutsche Schweiz. GV stimmt pro Altersfreitod. 24.5.2014. www.exit.ch/news/news/details/kommen-sie-zur-gv/ Kommentar dazu: Vögeli D. Alterssuizid erleichtern? Exits Spiel mit dem Feuer. Neue Zürcher Zeitung, 17.5.2014. www.nzz.ch/ zuerich/kommentare/1.18304153

2 Kierkegaard S. Synspunkter for min Forfatter Virksomhet (Der Gesichtspunkt für meine Wirksamkeit als Schriftsteller). In: Die Schriften über sich selbst. Regensburg: Eugen Diederichs Verlag; 1951. S. 38-9.

3 ASSM. Etude de l'ASSM «Attitude du corps médical face à l'assistance au suicide». Bull Méd Suisses. 2014;95(47):1767-9.

4 Borasio GD. Selbst bestimmt sterben. München: C.H. Beck; 2014, S. 104. Illustration modifiée tirée de: Gamondi C, Borasio GD, Limoni C, Preston N, Payne S. Legalisation of assisted suicide: a safeguard to euthanasia? Lancet. 2014;384:127. 